RESEARCHARTICLE

\title{
Effects of Fatigue, Driving Status, Cognition, and Depression on Participation in a Chronic Sample of Adults with Traumatic Brain Injury
}

\author{
Shannon B. Juengst ${ }^{1 *}$, Candice L. Osborne ${ }^{1}$, Kimberly S. Erler ${ }^{2}$, and Ketki D. Raina ${ }^{3}$ \\ ${ }^{1 *}$ Department of Physical Medicine \& Rehabilitation, University of Texas Southwestern Medical \\ Center, USA \\ ${ }^{2}$ Department of Occupational Therapy, Massachusetts General Hospital Institute of Health \\ Professions, USA \\ ${ }^{3}$ School of Exercise and Rehabilitation Sciences, College of Health and Human Services, University \\ of Toledo, USA
}

\section{Abstract}

Individuals with traumatic brain injury (TBI) often experience long-term participation restrictions. Physical and cognitive function, depression, age, ability to drive, and fatigue may contribute to participation restrictions post-TBI. The main objective of this study was to examine whether the ability to drive and fatigue contribute to participation among community-dwelling adults with TBI, after controlling for the age, cognition, and depression. We also explored the effects of these factors in a subgroup of participants who reporting clinically significant fatigue. This cross-sectional cohort study $(n=64)$ included adults with moderate to severe TBI, ranging from 6 months to 38 years post-injury.We measured participation with the Participation Assessment with Recombined Tools, using Rasch-adjusted scoring. We measured physical and cognitive fatigue with the Raschadjusted scoring of the modified Fatigue Impact Scale. Participants self-identified their ability to drive (Yes/No). Hierarchical linear regressions revealed that driving status accounted for $4 \%$ of the variance in participation $\left(F_{1,59} \Delta=4.05, p=.049, \mathrm{R}_{\text {adj }}{ }^{2} \Delta=.038\right)$, and fatigue accounted for $2 \%$ of the variance in participation $\left(F_{1,57} \Delta=1.77, p=.179, R_{\text {adj }}{ }^{2} \Delta=.019\right)$, after adjusting for age, depression, and cognition. The reverse was shown in the subgroup with clinically significant fatigue $(n=31)$; driving status accounted for only $1 \%$ of the variance in participation $\left(\mathrm{F}_{1,26} \Delta=0.71, p=.407, \mathrm{R}_{\mathrm{adj}}{ }^{2} \Delta=.009\right)$, whereas fatigue accounted for $15 \%$ of the variance in participation $\left(F_{124} \Delta=3.91, p=.034, R_{\text {adj }}{ }^{2} \Delta=.150\right)$. However, only physical fatigue, not cognitive fatigue, was a statistically significant independent predictor of participation. Depression, cognition, and ability to drive uniquely contributed to overall participation post-TBI. Among those with significant fatigue, depression and physical fatigue were the only significant predictors. The influence of personal and clinical factors on participation post-TBI may differ based on clinical symptoms an individual is experiencing. Personalized rehabilitation interventions selected based on clinical symptoms and targeting these modifiable factors could improve participation and quality of life for individuals with TBI.

This is an open access article distributed under the terms of the Creative Commons Attribution License, which permits unrestricted use, distribution, and reproduction in any medium, provided the original author and source are credited.

\section{Corresponding author:}

Shannon B. Juengst, Department of Physical Medicine \& Rehabilitation, University of Texas Southwestern Medical Center, USA

E-mail: Shannon.Juengst@UTSouthwestern.edu

\section{Keywords}

Traumatic brain injury, fatigue, participation, cognition, depression, driving

\section{Introduction}

The internationally accepted definition of "participation", defined by the World Health Organization's International Classification of Functioning, Disability and Health, is "involvement in a life situation". "Participation Restrictions" are "problems an individual may 
experience in involvement in life situations." Such broad definitions leave the concept of participation open for interpretation and dependent upon one's perspective. ${ }^{2}$ A synthesis of literature on the topic suggests that participation is characterized by "the extent to which social roles are fulfilled". ${ }^{3}$ Investigators developed several instruments in an attempt to further operationalize the concept of participation. While these instruments differ by content and measurement scale, most contain three common domains: productivity, social relationships, and community involvement. ${ }^{2}$ Therefore, we measured participation in community-dwelling individuals with traumatic brain injury ( $\mathrm{TBI}$ ) using the Participation Assessment with Recombined Tools-Objective (PART-O), a tool designed to examine long-term outcomes covering the most common domains of participation in individuals with TBI. ${ }^{2}$

Individuals with moderate to severe TBI experience long-term participation restrictions, including decreased social functioning. ${ }^{4-6}$ Participation is fundamental to the health and quality of life of individuals with TBI and considered an especially meaningful aspect of daily life by individuals with TBI, their family members, and the members of their healthcare team. ${ }^{7-9}$ Therefore, one of the primary goals of rehabilitation after TBI, and a prevailing indicator of successful functioning, is return to meaningful participation..$^{10,1}$ In order to accomplish this goal, an understanding of the areas that most commonly impact one's ability to engage in life situations after TBI is a crucial component of rehabilitation practice.

Previous literature identified many factors that contribute to participation after TBI, including physical and cognitive function, depression, age, ability to drive, and fatigue. Higher levels of functional independence, as measured by the FIM total and FIM subscale scores, were shown to be predictive of more participation up to 5 years after TBI. ${ }^{8,11,12}$ Individuals with depression or low positive affect (a hallmark of depression) demonstrated worse participation up to and after 5 years post-TBI. ${ }^{12-14}$ A recent investigation found that older age at time of injury predicted the trajectory of participation over the first 5 years after TBI, such that those who were older had worse participation that declined more quickly over time than those who were younger. ${ }^{12}$ Older age has also been further associated with unemployment and inability to drive at 5 years postTBI. $^{15}$

Research has shown that driving cessation after TBI, even in the presence of access to alternative means of transportation, is associated with worse objective participation. ${ }^{16,17}$ In a large sample of community-dwelling adults 5 years post moderate to severe TBI, driving status uniquely contributed to participation and was a stronger predictor than cognitive or motor function (measured by the $\mathrm{FIM}^{\mathrm{TM}}$ ) or depression. ${ }^{18}$ Driving may be a fundamental component to participation in out of home activities. The constellation of potential cognitive, emotional, motor, or sensory impairments that can occur after TBI could limit the ability to safely execute the complex tasks required for driving. Novack and colleagues (2010) found that, at 5 years post moderate to severe TBI, only $53 \%$ of survivors of TBI return to driving. ${ }^{19}$ Of those individuals who returned to driving, less than half underwent professional evaluation of fitness to do so. ${ }^{20}$

Chronic pathological fatigue has also been identified as a distressing symptom among 50$80 \%$ of individuals with $\mathrm{TBI}^{21-24}$ Fatigue is distinct from other comorbid symptoms such as depression, pain, and disturbed sleep, and as such, interventions targeting these comorbid symptoms may not mitigate fatigue..$^{25-27}$ While fatigue independently contributes to selfreported disability after $\mathrm{TBI}^{28}$ and a small number of studies suggest it could impact participation, ${ }^{29-31}$ its relationship to participation remains unclear. One study suggests that fatigue may not affect the frequency or quantity of participation, ${ }^{23}$ but little is known about its impact on quality of participation. Additionally, fatigue is generally conceptualized and measured as a unidimensional construct. However, common fatigue measures having multiple subscales, including physical fatigue and cognitive fatigue,$^{32-35}$ indicating multidimensionality. Particularly after neurological injury like TBI, individuals may experience differing levels of physical versus cognitive fatigue, though no studies to date have examined the effects of these individual domains on participation after TBI. Thus, the effects of fatigue on participation after TBI require further study.

The aims of the current study were to examine the extent to which ability to drive and fatigue (physical fatigue and cognitive fatigue) independently contribute to participation among community-dwelling adults with TBI, after controlling for age, cognitive function, and depression status. An additional exploratory aim was to examine whether fatigue has a stronger effect on participation among individuals experiencing clinically significant fatigue. 
We hypothesized that both ability to drive and fatigue would independently contribute to participation after TBI and that fatigue would have a stronger effect on participation among those experiencing clinically significant fatigue.

\section{Methods}

\section{Design}

We collected data from a cross-sectional cohort study of adults who were living in the community with a history of moderate to severe TBI. The broader purpose of the study was to examine factors contributing to participation after TBI, so the present analyses falls under this scope.

\section{Participants}

A University Institutional Review Board approved all research procedures, and we obtained written informed consent from all participants or their identified proxies. Inclusion criteria were: 1) history of moderate to severe TBI; 2) $\geq 6$ months post-injury; 3) living in the community; 4)>18 years old. Moderate to severe TBI was defined as any injury acquired from an outside force to the head and resulting in a Glasgow Coma Scale (GCS) ${ }^{36}$ score of 3-15 with positive neuroradiologic findings or sufficient functional compromise to require ongoing rehabilitative services. Exclusion criteria were: 1 ) comorbid condition resulting in progressive cognitive decline (e.g. dementia); 2) untreated psychosis or bipolar disorder; 3) current involvement in injury-related litigation.

\section{Recruitment and Screening}

We recruited participants through various sources, including: 1) the University of Pittsburgh's Rehabilitation Institute Research Registry; 2) existing research studies in the Departments of Physical Medicine \& Rehabilitation and Occupational Therapy at the University of Pittsburgh; 3 ) local community and hospital-based support groups for individuals with TBI and their family members; 4 ) local organizations providing services to adults with TBI. We screened individuals who expressed interest in participation for inclusion to confirm TBI diagnosis, review demographic information, and for untreated mental health conditions. We confirmed TBI diagnosis through medical record review or confirmed participation in ongoing specialty rehabilitation service when medical records were not available, and we screened for psychosis and bipolar disorder using the Mood Primary Care Evaluation of Mental Disorders (PRIME-MD). ${ }^{37}$

\section{Measures}

The first author collected all measures, meeting with participants in person at their place of residence or at a clinical facility of their choosing. Collected data included demographics (age, gender, race, education, driving status), injury-related characteristics (time since injury, injury severity), and clinical outcomes (functional disability, cognition, depression, fatigue impact, and participation).

Participation was measured using the Participation Assessment with Recombined Tools Objective (PART-O), a National Institute of Neurologic Disorders and Stroke Common Data Element for Participation after TBI. ${ }^{38}$ The PART-O measures frequency of participation in activities in the home and community, including items related to productivity, social interaction and relationships, and getting out of the home. . $^{238,39}$ The present study used the Raschadjusted PART-O score derived from 11 items of the PART-O and representing a unidimensional scale for participation. ${ }^{40}$ Scores range from 0-100.

Fatigue was measured using the modified Fatigue Impact Scale (mFIS), a tool that measures the impact of fatigue on everyday life in three dimensions that has been validated for use after $\mathrm{TBI}^{32,33}$ The mFIS includes 21 items rated on a 5-point ordinal scale $(0=$ no problem, $4=$ =xtreme problem) with references to the impact of fatigue over the past 4 weeks. Total scores range from $0-84$, with higher scores indicative of greater fatigue. Scores $>38$ indicate clinically significant fatigue. ${ }^{41}$ Rasch analysis of the mFIS in a sample with multiple sclerosis concluded that items represented two dimensions of fatigue: physical (8 items) and cognitive (5 items). ${ }^{34}$ We used an mFIS score $>38$ to determine participants for our subgroup analysis and used the Rasch-based Physical and Cognitive Fatigue scores to measure fatigue in our analyses.

Driving Status was measured through a single, self-reported question about whether or not 
participants were currently able to drive.

Depression status was determined using the Patient Health Questionnaire (PHQ9), which assesses the nine diagnostic criteria for major depressive disorder. ${ }^{37}$ Participants responded Yes or No to presence of each criterion nearly every day over the past two weeks. Participants were classified as having current depression if they endorsed one of the hallmark symptoms (e.g. depressed mood, lack of interest) and a minimum of 5 total symptoms. ${ }^{42}$ The PHQ9 is validated in moderate to severe TBI and is able to distinguish major depressive symptoms from the somatic and cognitive symptoms shared by both depression and TBI. ${ }^{42,43}$

Functional Disability was measured with the Mayo Portland Adaptability Inventory (MPAIIV), a 25-item measure of disability that captures abilities, adjustment, and participation. ${ }^{44,45}$ Items are rated on a $0-4$-point ordinal scale $(0=$ no problem, $4=$ severe problem), and a t-score (mean $=50, S D=10$ ) is derived from the total score. Higher $t$-scores indicate greater disability.

Cognitive function was measured using a composite $t$-score derived from a brief neuropsychological test batter validated within the TBI Model Systems national database study. ${ }^{46}$

This battery provided an overview of cognition in the cognitive domains most commonly affect by TBI. We included six of the eight neuropsychological tests from the validated battery, deemed most appropriate for a community-based sample. We calculated an overall Cognitive composite $t$-score by averaging relevant $t$-scores from each test, summarized below.

California Verbal Learning Test II (CVLT-II) is a 16-item list-learning task that incorporates learning, short-term delayed recall, long-term delayed recall, and long-term delayed recognition. ${ }^{47}$ The adjusted $t$-score for learning (total trials $1-5$ ) contributed to the Cognitive Composite score in this study.

Trail Making Test (TMT) is a timed test to measure visuoconceptual and visuomotor tracking. ${ }^{48}$ Participants connect sequential dots of numbers (Trails A) and alternating numbers and letters (Trails B) as quickly as they correctly can. Times to complete Trails A and Trails B were each converted to adjusted $t$-scores based on published norms, and both contributed to the Cognitive Composite $t$-score.

Symbol Digits Modalities test (SDMT) is a brief measure of visual perceptual skills, scanning, and psychomotor speed..$^{49}$ The total written score on the SDMT was converted to an adjusted t-score and used in the Cognitive Composite $t$-score.

Letter and Category Fluency (FAS/Animal Naming) assesses generativity and executive functioning by asking participants to name as many words as they can that begin with a certain letter (F-A-S) and to name as many different animals as they can (Animal Naming) in 60 seconds. ${ }^{50}$ Total fluency scores for FAS and for Animal Naming were converted to adjusted $t$ scores based on published norms, and both contributed to the Cognitive Composite score.

Wisconsin Cart Sorting Test (WCST) 64-item version assesses executive functions through a card sorting activity that requires cognitive flexibility and problem solving. ${ }^{51,52}$ The adjusted $t$ score for total perseverative errors contributed to the Cognitive Composite score.

\section{Analyses}

We analyzed data with SPSS 24.0 for Windows. First, we examined descriptive statistics to characterize the sample. Next, we conducted Spearman correlations to demonstrate relationships among our variables of interest. Last, we conducted three consecutive linear regressions (e.g. hierarchal regressions) to test our hypotheses that driving status and fatigue would each independently contribute to participation after controlling for cognitive impairment, depression status, and age. The first model (Model 1) included all covariates except driving status and fatigue. The second regression (Model 2) added driving status to the model. The final regression (Model 3 ) added fatigue (mFIS Physical and Cognitive subscales) to the model. The change in $\mathrm{R}^{2}$ values between each model represented the individual contribution of driving status and fatigue to participation, after controlling for the other covariates. We assessed model diagnostics to ensure good model fit, including variance inflation factors and tolerance.

To address our exploratory aim, we ran the hierarchical linear regressions again, this time only in a sample of participants with clinically significant fatigue. Again, the change in $\mathrm{R}^{2}$ values between each model represented the individual contribution of driving status and fatigue to participation, after controlling for the other covariates, and we assessed the same 
model diagnostics to ensure good model fit.

\section{Results}

\section{Descriptive}

Sixty-four participants completed the study. Table 1 summarizes the descriptive data for this study. For the full cohort, time since injury ranged from 6 months to 38 years, with a median time of $\sim 5.5$ years. Age ranged from 18 to 86 years old, and education ranged from 10 years (e.g. some high school) to 20 years (e.g. PhD or MD). Participants reported a mean standardized disability score within an average range, with a trend towards less disability than average $(M=44.3, S D=13.1)$. The mean fatigue score on the $\mathrm{mFIS}$ fell just below the threshold for clinically significant fatigue,$^{41}$ though it ranged from no fatigue (0) to severe fatigue (77). Cognition showed a similarly wide range, with $t$-scores as low as -1.3 and as high as $59.2(<40$ indicates cognitive impairment), though on average were within the range for normal cognition. Thirty-one participants (48\%) reported clinically significant fatigue. As expected, the subgroup reported more fatigue than the full cohort, and also reported slightly more disability and depression. All other factors were generally comparable.

\section{Correlations}

Table 2 reports the Spearman correlation coefficients for all variables. Rasch-adjusted PART-O scores were significantly correlated ( $p$ 's<.05) Physical fatigue, Cognition, Depression status, and Driving Status. Cognitive fatigue did not significantly correlate with the PART-O, though Cognitive fatigue and Physical fatigue were highly correlated ( $r=.809$, $\mathrm{p}<.01)$.

Table 1. Participant Characteristics

\begin{tabular}{|c|c|c|}
\hline & $\begin{array}{l}\text { Full Cohort } \\
\qquad(n=64) \\
\text { Mean ( } \pm \text { SD) }\end{array}$ & $\begin{array}{l}\text { Fatigue Subgroup } \\
\qquad \begin{array}{c}(n=31)^{\star} \\
\text { Mean }( \pm S D)\end{array}\end{array}$ \\
\hline Age (years) & $45.9( \pm 16.5)$ & $49.3( \pm 15.8)$ \\
\hline Time Since Injury (months) & $55.0(13.5-104.5)^{\dagger}$ & $59.0(14.0-148.0)^{\dagger}$ \\
\hline Education (years) & $14.6( \pm 2.6)$ & $14.3( \pm 2.4)$ \\
\hline Disability - MPAI (t-score) $)^{\ddagger}$ & $44.3( \pm 13.1)$ & $51.4( \pm 6.9)$ \\
\hline Fatigue $-\mathrm{mFIS}$ (total) $^{\ddagger}$ & $36.0( \pm 21.1)$ & $54.3( \pm 9.9)$ \\
\hline Physical Fatigue (mFIS subscale) $)^{\ddagger^{* *}}$ & $14.4(9.5)$ & $22.4(4.1)$ \\
\hline Cognitive Fatigue (mFIS subscale) $)^{\ddagger^{* *}}$ & $8.1(5.3)$ & $12.3(3.5)$ \\
\hline Cognitive Composite score (t-score) & $44.5( \pm 12.0)$ & $44.2( \pm 7.3)$ \\
\hline \multirow[t]{2}{*}{ Participation (PART-O)** } & $56.2( \pm 5.9)$ & $55.3( \pm 5.2)$ \\
\hline & n (\%) & n (\%) \\
\hline Gender (male) & $51(80 \%)$ & $23(74 \%)$ \\
\hline Race (white) & $62(97 \%)$ & $30(97 \%)$ \\
\hline Ability to drive (yes) & $44(69 \%)$ & $22(71 \%)$ \\
\hline \multicolumn{3}{|l|}{ Injury Severity } \\
\hline Moderate & $33(51 \%)$ & $16(51 \%)$ \\
\hline Severe & $31(48 \%)$ & $15(48 \%)$ \\
\hline Depression status (current depression) & $9(14 \%)$ & $6(19 \%)$ \\
\hline
\end{tabular}

* Subgroup: Participants with significant fatigue (mFIS $>38)$; † Median and interquartile range; † Higher scores indicate poorer outcomes; ${ }^{*}$ Scores based on Rasch analysis of measurement tool; MPAI=Mayo Portland Adaptability Inventory; mFIS=modified Fatigue Impact Scale; PART-O=Participation Assessment with Recombined Tools Objective 
Table 2. Spearman Correlations $(n=64)$

\begin{tabular}{|c|c|c|c|c|c|c|}
\hline & $\begin{array}{l}\text { Physical } \\
\text { Fatigue }\end{array}$ & $\begin{array}{l}\text { Cognitive } \\
\text { Fatigue }\end{array}$ & Cognition & $\begin{array}{l}\text { Depression } \\
\text { Status }\end{array}$ & $\begin{array}{l}\text { Driving } \\
\text { Status }\end{array}$ & $\begin{array}{l}\text { Age } \\
\text { Group }\end{array}$ \\
\hline $\begin{array}{l}\text { Participation (Rasch-adjusted PART- } \\
\text { O) }\end{array}$ & $-.260^{*}$ & -.174 & $.270^{*}$ & $-.299^{*}$ & $.304^{*}$ & -.110 \\
\hline Physical fatigue (mFIS subscale) & & $.809^{\dagger}$ & -.082 & .226 & -.005 & .224 \\
\hline Cognitive fatigue ( $\mathrm{mFIS}$ subscale) & & & -.099 & .177 & .060 & .040 \\
\hline $\begin{array}{l}\text { Cognition (Cognitive Composite T- } \\
\text { score) }\end{array}$ & & & & .026 & $.402^{\dagger}$ & .037 \\
\hline Depression status $(\mathrm{Y} / \mathrm{N})$ & & & & & -.022 & -.105 \\
\hline Driving status $(\mathrm{Y} / \mathrm{N})$ & & & & & & .028 \\
\hline Age Group & & & & & & \\
\hline
\end{tabular}

Table 3.Hierarchical Linear Regression Analyses for the Effects of Driving and Fatigue on Participation $(n=64)$

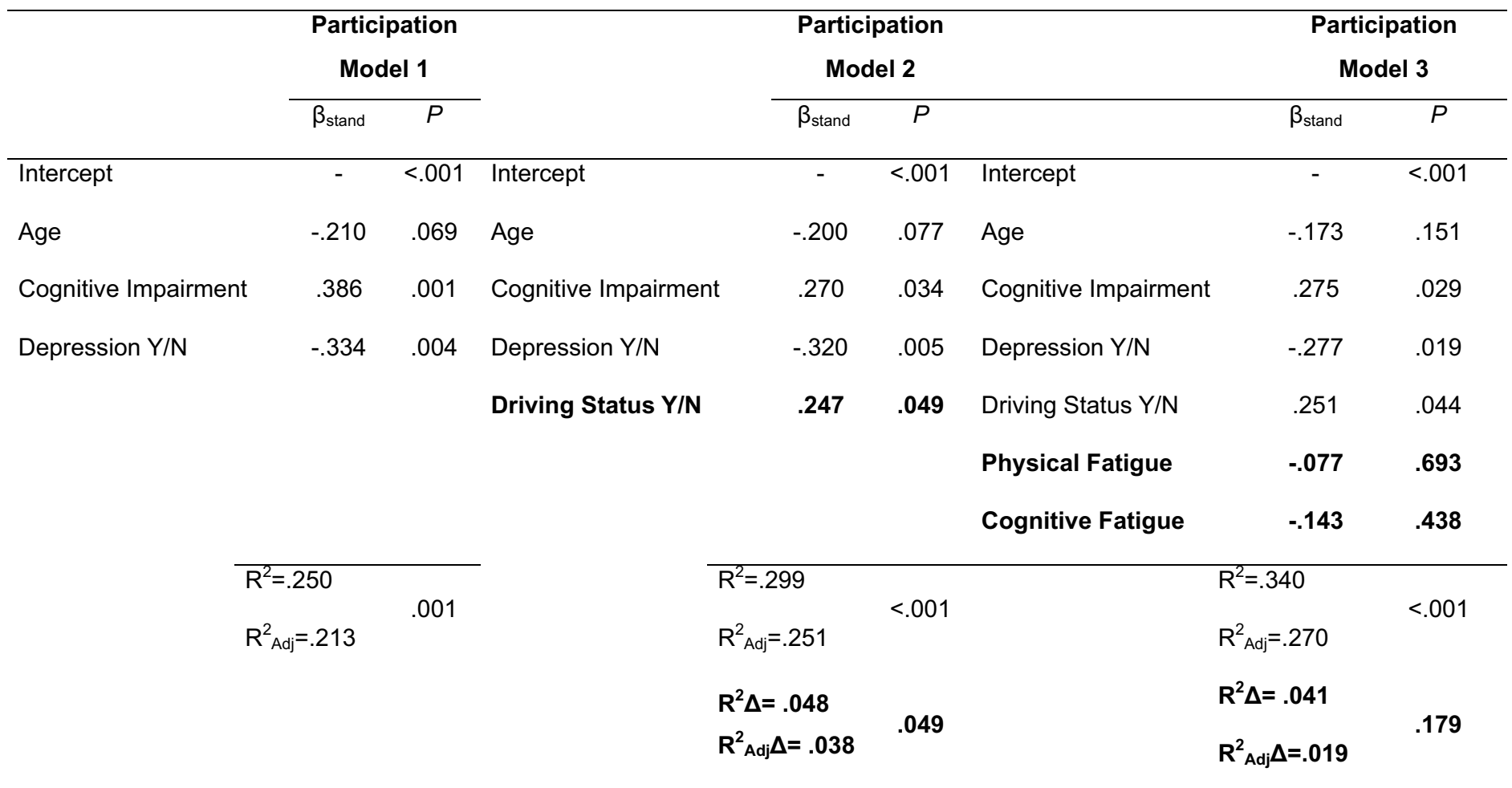

Note. $\mathrm{R}^{2} \Delta$ for Driving Status $\left(\mathrm{F}_{1,59} \Delta=4.046, P=0.049\right) ; \mathrm{R}^{2} \Delta$ for Physical and Cognitive Fatigue $\left(\mathrm{F}_{1,57} \Delta=1.772, P=0.179\right)$; . "No" is referent group for depression and driving status

\section{Hierarchical Linear Regressions}

Results from the primary regression analyses are summarized in Table 3. Overall, fatigue, driving status, cognition, depression, and age together explained $27 \%$ of the variance in participation among adults with TBI $\left(\mathrm{F}_{6,57}=4.89, p<.001, \mathrm{R}_{\mathrm{adj}}{ }^{2}=.270\right)$. Driving status alone accounted for $4 \%$ of the variance in participation $\left(\mathrm{F}_{1,59} \Delta=4.05, p=.049, \mathrm{R}_{\mathrm{adj}}{ }^{2} \Delta=.038\right)$ based on the change in the $R_{\text {adj }}{ }^{2}$ value from Model 1 (without driving or fatigue as covariates) to Model 2 
(with driving added as a covariate). Fatigue alone accounted for $2 \%$ of the variance in participation $\left(\mathrm{F}_{1,57} \Delta=1.77, p=.179, \mathrm{R}_{\mathrm{adj}}{ }^{2} \Delta=.019\right)$ based on the change in the $\mathrm{R}_{\mathrm{adj}}{ }^{2}$ value from Model 2 (without fatigue as covariates) to Model 2 (with fatigue added as a covariate). However, fatigue did not meet the threshold for statistical significance.

Based on the standardized betas in Model 3, cognition and depression $(|\beta| s=.28)$ were the strongest predictors of participation, followed by ability to drive $(\beta=.25)$. With regard to model diagnostics, the variance inflation factor ranged from 1.12 to 3.25 , indicating moderate correlations among covariates, and the tolerance ranged from .30 to .89 , indicating no problem with multicollinearity.

\section{Exploratory Analyses: Subgroup of Participants with Clinically Significant Fatigue}

Table 4 reports the Spearman correlation coefficients for all variables in the subgroup $(n=31)$. Rasch-adjusted PART-O scores were significantly correlated ( $p$ 's<.05) Physical fatigue and Depression status only. Unlike in the full cohort, Cognitive fatigue and Physical fatigue did not significantly correlate in the subgroup.

Table 5 summarizes results from the exploratory regression analyses. Overall, fatigue, driving status, cognition, depression, and age together explained $33 \%$ of the variance in participation among adults with clinically significant fatigue after $\mathrm{TBI}\left(\mathrm{F}_{6,24}=3.44, p=.013\right.$, $\left.\mathrm{R}_{\mathrm{adj}}{ }^{2}=.328\right)$. Driving status alone accounted for only $1 \%$ of the variance in participation $\left(\mathrm{F}_{1,26} \Delta=0.71, p=.407, \mathrm{R}_{\mathrm{adj}}{ }^{2} \Delta=.009\right)$ and was not statistically significant. Fatigue alone accounted for $15 \%$ of the variance in participation $\left(\mathrm{F}_{124} \Delta=3.91, p=.034, \mathrm{R}_{\mathrm{adj}}{ }^{2} \Delta=.150\right)$. Based on the standardized betas in Model 3, Depression ( $\beta=-.39)$ and Physical fatigue $(\beta=-.38)$ were the strongest predictors of participation. Though fatigue overall contributed $15 \%$ of the variance in participation, only Physical fatigue, and not Cognitive fatigue, was a statistically significant independent predictor of participation. With regard to model diagnostics, the variance inflation factor ranged from 1.27 to 1.53 , indicating low moderate correlations among covariates, and the tolerance ranged from .67 to .84 , indicating no problem with multicollinearity.

Table 4. Spearman Correlations in Subgroup with Clinically Significant Fatigue $(n=31)$

\begin{tabular}{|c|c|c|c|c|c|c|}
\hline & $\begin{array}{l}\text { Physical } \\
\text { Fatigue }\end{array}$ & $\begin{array}{c}\text { Cognitive } \\
\text { Fatigue }\end{array}$ & Cognition & $\begin{array}{c}\text { Depression } \\
\text { Status }\end{array}$ & $\begin{array}{l}\text { Driving } \\
\text { Status }\end{array}$ & Age Group \\
\hline Participation (Rasch-adjusted PART-O) & $-.458 *$ & -.262 & .125 & $-.471 \dagger$ & .169 & .244 \\
\hline Physical fatigue (mFIS subscale) & & .311 & .201 & .165 & -.092 & -.110 \\
\hline Cognitive fatigue (mFIS subscale) & & & -.038 & .032 & .044 & $-.366^{*}$ \\
\hline Cognition (Cognitive Composite T-score) & & & & .164 & $.461^{\dagger}$ & .120 \\
\hline Depression status $(\mathrm{Y} / \mathrm{N})$ & & & & & -.046 & -.242 \\
\hline Driving status $(\mathrm{Y} / \mathrm{N})$ & & & & & & .281 \\
\hline Age Group & & & & & & \\
\hline
\end{tabular}

*mFIS subscales are based on Rasch-adjusted scales. The Physical fatigue subscales contains the two items on the original psychosocial subscale of the mFIS. Age groups are 18-24, 24-59, 60+ years old. ${ }^{*} p<.05 ; \dagger p<.01 ; 2$-tailed correlations 
Table 5. Hierarchical Linear Regression Analyses for the Effects of Driving and Fatigue on Participation in Subgroup with Clinically Significant Fatigue $(n=31)$

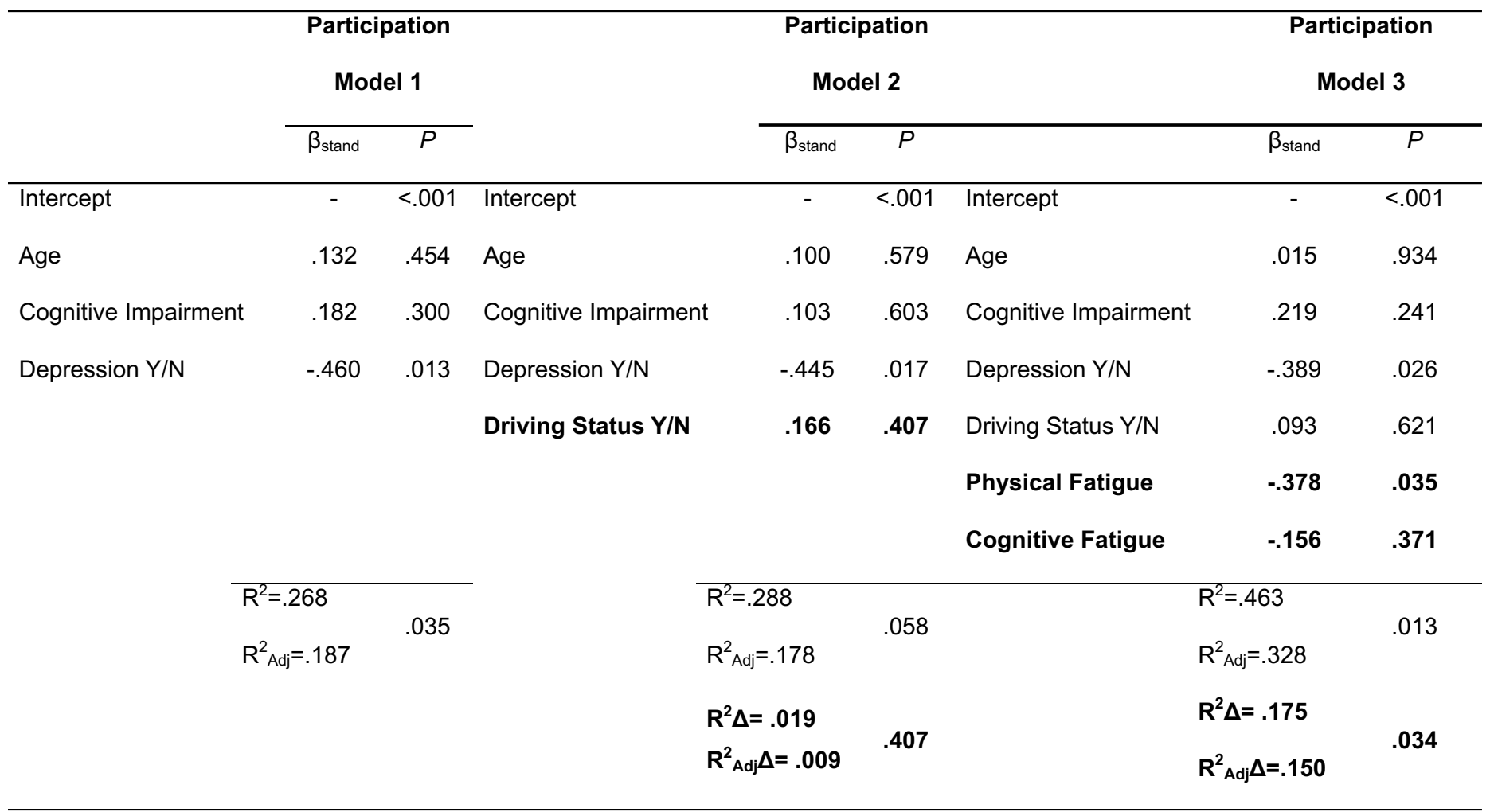

Note. $\mathrm{R}^{2} \Delta$ for Driving Status $\left(\mathrm{F}_{1,26} \Delta=0.710, P=0.407\right) ; \mathrm{R}^{2} \Delta$ for Physical and Cognitive Fatigue $\left(\mathrm{F}_{1,24} \Delta=3.909, P=0.034\right) ;$. "No" is referent group for depression and driving status

\section{Discussion}

This investigation into the independent contributions of driving ability and fatigue on participation after TBI corroborates the small body of previous literature on post-TBI participation, but also raises new questions as to the ability to apply findings of this nature to individuals with TBI as a whole. Our work supports the effect of driving on participation and the idea that fatigue does not contribute to frequency of participation in community-based activities. However, we found the reverse when looking only in a subgroup of individuals with TBI who are experiencing notable fatigue. Driving did not influence participation, and Physical fatigue was one of the strongest contributing factors. This suggests that the influence of personal and clinical factors on participation post-TBI may differ based on clinical symptoms an individual may be experiencing.

Although more than half of individuals with TBI return to driving by 5 years post injury, there is limited literature examining the association of driving with participation. ${ }^{19}$ The few studies that exist have found that individuals who drive have better participation and social outcomes than non-drivers. ${ }^{16-18}$ This study provides further evidence that individuals who return to driving after TBI have a higher frequency of participation. The items on the PART-O that involve activities happening outside of the home (e.g. eating in restaurants, going to the movies, or shopping) are those most likely to occur more frequently as a result of driving. Interestingly, driving was not a significant contributor to participation for individuals with TBI who were also experiencing notable fatigue. Perhaps, these individuals are distinct in that they have been forced to develop additional coping and compensatory strategies to complete tasks outside of the home in the setting of clinically significant fatigue. These individuals may include a prioritization system as a part of energy conservation techniques such as choosing to shop online instead of a store in order to be able to have the energy to attend religious activities outside of the home. Although the Rasch-adjusted PART-O scores for this subgroup were similar to those without clinically significant fatigue, another possible explanation is that they are participating more in activities that occur within the home and limiting activities outside of the home, while those without clinically significant fatigue may have more of a balance 
between in and out of home items. Future research should compare within the home versus outside the home participation between those with clinically significant fatigue and those without it to test this hypothesis. While one scoring algorithm for the PART-O has a subscale for "Out and About", ${ }^{2}$ there is no comparative subscale for within the home participation. Future studies should also examine the association of driving status with quality of participation and satisfaction with participation.

Only a small body of evidence exists for the impact of fatigue on participation after TBI. Among adults with chronic TBI, fatigue independently predicted disability, ${ }^{28}$ as measured by the MPAI-IV, which does contain a validated subscale for participation. ${ }^{44}$ However, this small study did not investigate participation specifically. Another study found no association between fatigue and frequency of participation post-TBI, but did find that fatigue was highly associated with quality of life..$^{23}$ This led the authors to suggest that fatigue may influence quality of participation, rather than frequency of participation in major life activities. ${ }^{23}$ In our study, we measured participation using the PART-O, which measures frequency of participation and not quality of participation. For example, individuals may report that they are engaging in homemaking activities 35 or more hours per week (a high frequency of participation), but compared to before injury when they were preparing full course meals and cleaning the house every day, they are now only preparing cold sandwiches and cleaning one room of the house each day. Similarly, individuals may report that they socialize with friends 10-19 times per week, but this does not differentiate whether they are having long, meaningful conversations with friends or whether they are only able to socialize for 20 minutes at a time in order to effectively conserve energy.

Our study does suggest that, among those experiencing high levels of fatigue, their Physical fatigue (but not cognitive fatigue) may influence their frequency of participation. At a certain point, physical fatigue may put a ceiling on the number or amount of certain activities that a person can accomplish or engage in. Cognitive fatigue did not show a similar relationship; it could be that individuals may continue to be able to engage in certain activities despite cognitive fatigue, though again, the quality of their engagement may diminish as a result. It is possible that individuals experiencing significant fatigue do not differentiate well between physical and cognitive fatigue, hence perceiving and reporting the effects of fatigue on physical activities and not the effects of fatigue on cognitive functions. Overall, the impact of fatigue on participation may differ based on severity and nature (e.g. physical, cognitive) of the fatigue experienced and by dimension of participation (e.g. frequency, quality, satisfaction).

This study supports the growing body of literature post-TBI indicating that frequency, quality, and satisfaction are distinct dimensions of participation. ${ }^{14,23,53-55}$ The notion that participation is multi-dimensional is consistent with the theoretical foundation of the ICF framework. Complex interactions between ICF components are not always a predictable one-to-one relationship and influence an individual's experiences and perception of those experiences. A person's physical, mental, emotional, and environmental characteristics are perpetually interacting in new ways, which continuously alters ability and/or desire to participate in the surrounding world. ${ }^{56}$ The results of this study contribute to a more comprehensive understanding of the complexities of participation after TBI, specifically the influences of driving and fatigue, as defined by the ICF theoretical model.

The strongest predictors of participation among adults with chronic TBI in our study were cognitive impairment and depression. This is consistent with previous studies on participation after TBI. ${ }^{5,12,14,57,58}$ Of note, however, is that among those with high levels of fatigue, cognitive impairment was not associated with participation, despite comparable cognitive composite and participation scores between the full cohort and the subgroup with high fatigue. In these individuals, the experience of fatigue - particularly physical fatigue - may be so distressing that it replaces cognitive impairment as the predictor of frequency of participation. This is notable as it has implications for intervention. First, measuring and addressing fatigue as a multidimensional construct (e.g. looking at both physical and cognitive fatigue separately and in conjunction) may yield a more complete and specific characterization of an individual to inform intervention. Second, for individuals with significant chronic fatigue after TBI, management of fatigue should be the primary target of intervention.

\section{Study Limitations}

This study was a secondary analysis of existing data from a cross-sectional cohort study. 
Participants were not representative of all community-dwelling adults with a history of TBI, particularly with regard to racial and ethnic diversity. Men were also over-represented in this sample, even given the higher percentage of men among those sustaining a TBI. The measures used in this study, other than for cognition, were self-reported, so recall bias or poor selfawareness may have influenced the study results. However, despite the limitations of selfreported measures, it is still the most feasible - and sometimes the most appropriate - method of data collection for studies answering questions of this nature. Development of a cut-off score for clinically significant fatigue in a moderate to severe TBI sample, particularly using the Raschadjusted mFIS subscale scores (e.g. physical fatigue, cognitive fatigue) is warranted. The heterogeneity of the sample, with regard to age, time since injury, cognition, fatigue, and participation, was both a strength and a limitation. It reduced our statistical power while simultaneously increasing our ability to generalize more broadly to the TBI population. Large longitudinal studies would be better able to examine the effects of chronicity of injury and age, and the interplay between the two, on participation. Though challenging in a community-based sample, it would be beneficial to have a more thorough characterization of the initial injury, including loss of consciousness, length of post-traumatic amnesia, location of injury, extracranial injuries, etc. Finally, we looked only at driving status and not more broadly at independent transportation. Future research should examine these relationships in a larger heterogeneous sample, with better racial, ethnic, gender, and geographic diversity.

\section{Conclusions}

Depression, cognition, and ability to drive uniquely contribute to overall participation among community-dwelling adults with a history of moderate to severe TBI. Among the subgroup with clinically significant fatigue, depression and physical fatigue are the only significant predictors. This suggests that the influence of personal and clinical factors on participation post-TBI may differ based on clinical symptoms an individual may be experiencing. Personalized rehabilitation interventions, selected based on clinical symptoms and targeting these potentially modifiable factors, could improve participation and subsequently improve quality of life for individuals with TBI.

\section{Funding}

This study was supported in part through funding received from the School of Health and Rehabilitation Science Research Development Fund, School and Health of Rehabilitation Science, University of Pittsburgh (SJ); by the National Institutes of Health Clinical Loan Repayment Program NICHD L30 NS089099-02 (SJ), and by the U.S. Army Medical Research and Material Command under Award No. W81XWH-10-1-0920 (KR, SJ). Opinions, interpretations, conclusions, and recommendations are those of the author and are not necessarily endorsed by the U.S. Army. 


\section{References}

1. Organization WH. International Classification of Functioning, Disability and Health: ICF. World Health Organization; 2001.

2. Bogner JA, Whiteneck GG, Corrigan JD, et al. Comparison of scoring methods for the participation assessment with recombined tools-objective. Arch Phys Med Rehabil. 2011;92(4):552-563. doi:10.1016/j.apmr.2010.11.014.

3. Whiteneck G, Dijkers MP. Difficult to measure constructs: conceptual and methodological issues concerning participation and environmental factors. Arch Phys Med Rehabil. 2009;90(11 Suppl):S22-S35. doi:10.1016/j.apmr.2009.06.009.

4. Temkin NR, Corrigan JD, Dikmen SS, Machamer J. Social functioning after traumatic brain injury. J Head Trauma Rehabil. 2009;24(6):460-467. doi:10.1097/HTR.0b013e3181c13413.

5. Larsson J, Björkdahl A, Esbjörnsson E, Sunnerhagen KS. Factors affecting participation after traumatic brain injury. J Rehabil Med Off J UEMS Eur Board Phys Rehabil Med. 2013;45(8):765-770. doi:10.2340/16501977-1184.

6. Sander AM, Krentzer JS, Rosenthal M, Delmonico R, Young ME. A Multicenter Longitudinal Investigation of Return to Work and Community Integration Following Traumatic Brain Injury. $J$ Head Trauma Rehabil. 1996;11(5):70.

7. Williams MW, Rapport LJ, Millis SR, Hanks RA. Psychosocial outcomes after traumatic brain injury: Life satisfaction, community integration, and distress. Rehabil Psychol. 2014; 59(3):298-305. doi:10.1037/a0037164.

8. Huebner RA, Johnson K, Bennett CM, Schneck C. Community Participation and Quality of Life Outcomes After Adult Traumatic Brain Injury. Am J Occup Ther. 2003;57(2):177-185. doi:10 .5014/ajot.57.2.177.

9. Steadman-Pare D, Colantonio A, Ratcliff G, Chase S, Vernich L. Factors associated with perceived quality of life many years after traumatic brain injury. $J$ Head Trauma Rehabil. 2001;16(4):330-342.

10. Cicerone KD. Participation as an outcome of traumatic brain injury rehabilitation. $J$ Head Trauma Rehabil. 2004;19(6):494-501.

11. Erler K, Locascio J, Whiteneck G, et al. Predicting the Trajectories of Social Participation After Traumatic Brain Injury: A Longitudinal Analysis. Arch Phys Med Rehabil. 2016;97(12):e16. doi:10.1016/j.apmr.2016.09.042.

12. Erler K, Whiteneck GG, Juengst SB, et al. Predicting the trajectory of participation after traumatic brain injury: A longitudinal analysis. J Head Trauma Rehabil. 2017;Accepted for publication.

13. Hart T, Brenner L, Clark AN, et al. Major and minor depression after traumatic brain injury. Arch Phys Med Rehabil. 2011;92(8):1211-1219. doi:10.1016/j.apmr.2011.03.005.

14. Juengst SB, Arenth PM, Raina KD, McCue M, Skidmore ER. Affective State and Community Integration After Traumatic Brain Injury. Am J Phys Med Rehabil Assoc Acad Physiatr. 2014. doi:10.1097/PHM.0000000000000163.

15. Corrigan JD, Cuthbert JP, Harrison-Felix C, et al. US Population Estimates of Health and Social Outcomes 5 Years After Rehabilitation for Traumatic Brain Injury. J Head Trauma Rehabil. 2014. doi:10.1097/HTR.0000000000000020.

16. Rapport LJ, Hanks RA, Bryer RC. Barriers to driving and community integration after traumatic brain injury. J Head Trauma Rehabil. 2006;21(1):34-44.

17. Rapport LJ, Bryer RC, Hanks RA. Driving and community integration after traumatic brain injury. Arch Phys Med Rehabil. 2008;89(5):922-930. doi:10.1016/j.apmr.2008.01.009.

18. Erler K, Juengst SB, smith DL, et al. Examining driving and participation 5 years after traumatic brain injury. OJTR Occup Particip Health. 2017.

19. Novack TA, Labbe D, Grote M, et al. Return to driving within 5 years of moderate-severe traumatic brain injury. Brain Inj. 2010;24(3):464-471. doi:10.3109/02699051003601713.

20. Fisk GD, Schneider JJ, Novack TA. Driving following traumatic brain injury: prevalence, exposure, advice and evaluations. Brain Inj. 1998;12(8):683-695. 
21. Olver JH, Ponsford JL, Curran CA. Outcome following traumatic brain injury: a comparison between 2 and 5 years after injury. Brain Inj. 1996;10(11):841-848.

22.Ashman TA, Cantor JB, Gordon WA, et al. Objective measurement of fatigue following traumatic brain injury. J Head Trauma Rehabil. 2008;23(1):33-40. doi:10. 1097/01 .HTR. 0000308719.70288 .22 .

23. Cantor JB, Ashman T, Gordon W, et al. Fatigue After Traumatic Brain Injury and Its Impact on Participation and Quality of Life: J Head Trauma Rehabil. 2008;23(1):41-51. doi:10. 1097/ 01. HTR.0000308720.70288.af.

24.Borgaro SR, Gierok S, Caples H, Kwasnica C. Fatigue after brain injury: initial reliability study of the BNI Fatigue Scale. Brain Inj. 2004;18(7):685-690. doi:10.1080/02699050310001646080.

25. Bushnik T, Englander J, Wright J. The experience of fatigue in the first 2 years after moderate-tosevere traumatic brain injury: a preliminary report. J Head Trauma Rehabil. 2008;23(1):17-24. doi:10.1097/01.HTR.0000308717.80590.22.

26.Cantor JB, Ashman T, Bushnik T, et al. Systematic Review of Interventions for Fatigue After Traumatic Brain Injury: A NIDRR Traumatic Brain Injury Model Systems Study. J Head Trauma Rehabil. 2014;29(6):490-497. doi:10.1097/HTR.0000000000000102.

27.Beaulieu-Bonneau S, Ouellet M-C. Fatigue in the first year after traumatic brain injury: course, relationship with injury severity, and correlates. Neuropsychol Rehabil. 2016:1-19. doi:10. 1080/09602011.2016.1162176.

28.Juengst S, Skidmore E, Arenth PM, Niyonkuru C, Raina KD. Unique contribution of fatigue to disability in community-dwelling adults with traumatic brain injury. Arch Phys Med Rehabil. 2013;94(1):74-79. doi:10.1016/j.apmr.2012.07.025.

29.Lequerica AH, Botticello AL, Lengenfelder J, et al. Factors associated with remission of posttraumatic brain injury fatigue in the years following traumatic brain injury (TBI): a TBI model systems module study. Neuropsychol Rehabil. September 2016:1-12. doi:10.1080/09602011 .2016.1231120.

30.Sherer M, Davis LC, Sander AM, et al. Prognostic Importance of Self-reported Traits/Problems/Strengths and Environmental Barriers/facilitators for Predicting Community Integration and Participation Outcomes in Persons with Traumatic Brain Injury: A Systematic Review. Arch Phys Med Rehabil. 2014. doi:10.1016/j.apmr.2014.02.006.

31.Dumont C, Gervais M, Fougeyrollas P, Bertrand R. Toward an explanatory model of social participation for adults with traumatic brain injury. J Head Trauma Rehabil. 2004;19(6) : 431-444.

32.Larson RD. Psychometric properties of the modified fatigue impact scale. Int J MS Care. 2013;15(1):15-20. doi:10.7224/1537-2073.2012-019.

33. Schiehser DM, Delano-Wood L, JakAJ, et al. Validation of the Modified Fatigue Impact Scale in Mild to Moderate Traumatic Brain Injury. J Head Trauma Rehabil. 2014. doi:10.1097/HTR . 0000000000000019 .

34.Mills RJ, Young CA, Pallant JF, Tennant A. Rasch analysis of the Modified Fatigue Impact Scale (MFIS) in multiple sclerosis. J Neurol Neurosurg Psychiatry. 2010;81(9):1049-1051. doi:10. 1136/jnnp.2008.151340.

35. Shuman-Paretsky M, Zemon V, Foley FW, Holtzer R. Development and Validation of the StateTrait Inventory of Cognitive Fatigue in Community-Dwelling Older Adults. Arch Phys Med Rehabil. 2016. doi:10.1016/j.apmr.2016.07.024.

36. Teasdale G, Jennett B. Assessment of coma and impaired consciousness. A practical scale. Lancet. 1974;2(7872):81-84.

37.Spitzer RL, Kroenke K, Williams JB. Validation and utility of a self-report version of PRIME-MD: the PHQ primary care study. Primary Care Evaluation of Mental Disorders. Patient Health Questionnaire. JAMA.1999;282(18):1737-1744.

38. Whiteneck GG, Dijkers MP, Heinemann AW, et al. Development of the participation assessment with recombined tools-objective for use after traumatic brain injury. Arch Phys Med Rehabil. 2011;92(4):542-551. doi:10.1016/j.apmr.2010.08.002.

39.Bogner J, Bellon K, Kolakowsky-Hayner SA, Whiteneck G. Participation assessment with recombined tools-objective (PART-O). J Head Trauma Rehabil. 2013;28(4):337-339. doi:10.1097/HTR.0b013e31829af969. 
40. Malec JF, Whiteneck GG, Bogner JA. Another Look at the PART-O Using the Traumatic Brain Injury Model Systems National Database: Scoring to Optimize Psychometrics. Arch Phys Med Rehabil. 2016;97(2):211-217. doi:10.1016/j.apmr.2015.09.001.

41.Flachenecker $P$, Kümpfel T, Kallmann B, et al. Fatigue in multiple sclerosis: a comparison of different rating scales and correlation to clinical parameters. Mult Scler J. 2002;8(6):523-526.

42.Fann JR, Bombardier CH, Dikmen S, et al. Validity of the Patient Health Questionnaire-9 in assessing depression following traumatic brain injury. J Head Trauma Rehabil. 2005;20(6) :501-511.

43. Cook KF, Bombardier $\mathrm{CH}$, Bamer AM, et al. Do somatic and cognitive symptoms of traumatic brain injury confound depression screening? Arch Phys Med Rehabil. 2011;92(5):818-823. doi:10.1016/j.apmr.2010.12.008.

44. Malec JF. The Mayo-Portland Participation Index: A brief and psychometrically sound measure of brain injury outcome. Arch Phys Med Rehabil. 2004;85(12):1989-1996.

45. Malec JF, Kragness M, Evans RW, et al. Further psychometric evaluation and revision of the Mayo-Portland Adaptability Inventory in a national sample. J Head Trauma Rehabil. 2003;18(6):479-492.

46. Hanks RA, Millis SR, Ricker JH, et al. The predictive validity of a brief inpatient neuropsychologic battery for persons with traumatic brain injury. Arch Phys Med Rehabil. 2008; 89(5):950-957. doi:10.1016/j.apmr.2008.01.011.

47.Delis DC, Kramer JH, Kaplan E, Ober BA. CVLT-II. Psychol Corp. 2000.

48.Lezak: Neuropsychological assessment- Google Scholar. Retrieved from: https://scholar .google.com/ scholar_lookup? publication_year= 1983\&author=M.+D. +Lezak\&t itle=+Neuropsychological+asse-ssment

49.Smith A. Symbol digit modalities test (SDMT) manual (revised) Western Psychological Services. Los Angel. 1982.

50.Borkowski JG, Benton AL, Spreen O. Word fluency and brain damage. Neuropsychologia. 1967;5:135-140.

51.Anderson SW, Damasio H, Jones RD, Tranel D. Wisconsin Card Sorting Test Performance as a Measure of Frontal Lobe Damage. J Clin Exp Neuropsychol. 1991;13(6):909-922. doi:10. 1080/01688639108405107.

52.Kongs SK, Thompson LL, Iverson GL, Heaton RK. WCST-64: Wisconsin Card Sorting Test-64 Card Version, Professional Manual. PAR; 2000.

53. Cicerone KD, Azulay J. Perceived self-efficacy and life satisfaction after traumatic brain injury. $J$ Head Trauma Rehabil. 2007;22(5):257-266. doi:10.1097/01.HTR.0000290970.56130.81.

54. Hart T, Sherer M, Temkin N, et al. Participant-Proxy Agreement on Objective and Subjective Aspects of Societal Participation Following Traumatic Brain Injury: J Head Trauma Rehabil. 2010;25(5):339-348. doi:10.1097/HTR.0b013e3181c7e60b.

55. Sander AM, Clark A, Pappadis MR. What is community integration anyway?: defining meaning following traumatic brain injury. J Head Trauma Rehabil. 2010;25(2):121-127. doi:10.1097 /HTR.0b013e3181cd1635.

56.World Health Organization. World Wealth Organization International Statistical Classification of diseases and related health problems. In: 1992.

57.Eriksson G, Kottorp A, Borg J, Tham K. Relationship between occupational gaps in everyday life, depressive mood and life satisfaction after acquired brain injury. J Rehabil Med. 2009;41(3):187-194. doi:10.2340/16501977-0307.

58.Lewis FD, Horn GJ. Depression following traumatic brain injury: Impact on post-hospital residential rehabilitation outcomes. Neuro Rehabilitation. 2017. doi:10.3233/NRE-161427. 